\title{
LAND COVER CHANGE MONITORING OF TYPICAL FUNCTIONAL COMMUNITIES OF SICHUAN PROVINCE BASED ON ZY-3 DATA
}

\author{
G. M. $\mathrm{Li}^{1 \mathrm{a}} *, \mathrm{~S} . \mathrm{Li}^{1 \mathrm{~b}}, \mathrm{G} . \mathrm{W}$. Ying ${ }^{1 \mathrm{c}}, \mathrm{X} . \mathrm{P} . \mathrm{Wu}^{2}$ \\ ${ }^{1}$ The Sixth Landforms Surveying Team of NASMG, Chengdu, China \\ a*:105189857@qq.com; b:250970390@qq.com; c:331293996@qq.com \\ ${ }^{2}$ Sichuan Normal University, Chengdu, China-349100531@qq.com
}

Commission III, WG VI/7

KEY WORDS: Land Cover, ZY-3, Functional Communities, Sichuan Province

\begin{abstract}
:
According to the function, land space types are divided into key development areas, restricted development areas and forbidden development areas in Sichuan Province. This paper monitors and analyses the changes of land cover in different typical functional areas from 2010 to 2017, which based on ZY-3 high-score images data and combined with statistical yearbook and thematic data of Sichuan Province. The results show that: The land cover types of typical key development zones are mainly composed of cultivated land, forest land, garden land, and housing construction land, which accounts for the total area of land cover $87 \%$. The land cover types of typical restricted development zone mainly consists of forest land and grassland, which occupy $97.71 \%$ of the total area of the surface coverage. The land cover types of the typical prohibition development zone mainly consist of forest land, grassland, desert and bared earth, which accounts for the total area of land cover $99.31 \%$.
\end{abstract}

\section{INTRODUCTION}

Land space in Sichuan province is divided into key development zones, restricted development zone and prohibition development zone (Liu, 2016a and Fan, 2015a). The main functional areas is an important area protecting natural and cultural resources in Sichuan province, is important soil and water conservation area and important drinking water source protection area. Under the premise of strict protection of the ecological environment, rational develop advantages and characteristics of tourism resources, develop eco-tourism industry, control strictly the interference of human factors on the natural ecology, prohibit the development activities which does not comply with the main functional areas targeted, guide population transfer gradual and orderly, reach the achievement of pollutant "zero emissions", improve environmental quality, enhance capacity for sustainable development (Pei, 2010a).

This paper takes the main functional area of Sichuan Province as the research object, based on the ZY-3 image, combined with statistical yearbook and special data, to monitor the changes of surface coverage of key ecological function areas in Sichuan province, which is very important significance for us to learn about the current situation of regional ecological civilization, maintain regional ecological security, prompt the relationship between environment and economic development policies and promote the construction of the main functional area of the national construction pilot demonstration (Wang et al., 2017a).

\section{RESEARCH AREA OVERVIEW}

Key development zones include 89 counties (cities, districts) of the 19 cities (districts) in Chengdu plain, the southern of Sichuan, and the northeast of Sichuan, and the western of
Sichuan, as well as the connected 50 punctate development towns (Zhang et al., 2010a). Among them, Chengdu plain is a national level key development zone, which main function positioning is the important economic center in the West, the national comprehensive transportation hub, the business logistics center and the financial center, and a base of advanced manufacturing industry, scientific technological innovation industrial base and agricultural products processing base.

Restricted development zone includes ecological function areas and agricultural products producing areas. Among them, the national level key ecological function areas includes Ruoergai prairie wetland ecological function areas, Sichuan-yunnan forest biodiversity and ecological function areas, qinba biodiversity ecological function areas and others, a total of 25 regions. There is no doubt that such wide coverage is of rich biodiversity.

Prohibition development zone is an important protection area of natural and cultural resources, is also a key ecological function area needing special protection, which prohibits any other industrialization and urbanization development, including national level and provincial level two parts (Wang et al., 2017a and Sun et al., 2015a). The national prohibition development zone include national nature reserve, world cultural and natural heritage, national scenic area, national forest park, national important wetlands, national wetland park and national geological park; The provincial prohibition development zone include provincial and lower levels of various types of natural and cultural resource protection area, important drinking water sources, and other prohibited development area which to be determined by provincial People's Government (Nie et al., 2010a and Li et al., 2013). 


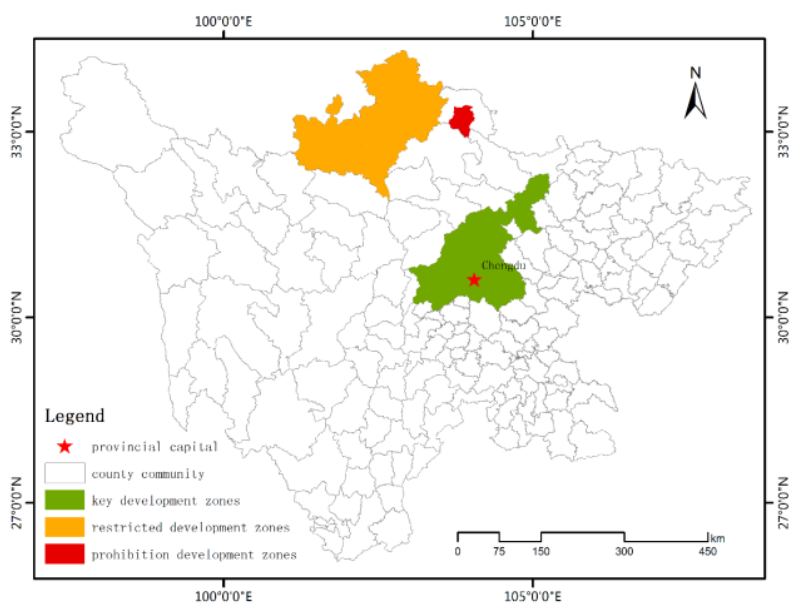

Figure 1. Regional location

\section{DATA AND METHOD}

\subsection{Data Source}

ZY-3 high resolution remote sensing image data are derived from the first national survey of national geographical conditions, DEM, DOM, 1:5 million scale, the administrative division and other data are derived from the Sichuan geo information bureau.

\subsection{Method}

Based on the ZY-3 orthographic image, surface coverage data production is performed by means of full manual acquisition or human-computer interaction interpretation.

\section{RESEARCH RESULT}

\begin{tabular}{|ccccc|}
\hline $\begin{array}{c}\text { Vegetation } \\
\text { Types }\end{array}$ & $\begin{array}{c}\text { Area } \\
\left(\mathrm{km}^{2}\right)\end{array}$ & $\begin{array}{c}\text { Proportion } \\
(\%)\end{array}$ & $\begin{array}{c}\text { Area } \\
\left(\mathrm{km}^{2}\right)\end{array}$ & $\begin{array}{c}2017 \\
\text { Proportion } \\
(\%)\end{array}$ \\
\hline $\begin{array}{c}\text { Cultivated } \\
\text { land }\end{array}$ & 9299.32 & 39.52 & 7858.38 & 33.40 \\
$\begin{array}{c}\text { Garden land } \\
\text { Forest land }\end{array}$ & 1743.39 & 7.41 & 2414.25 & 10.26 \\
$\begin{array}{c}\text { Grass land } \\
\text { Housing }\end{array}$ & 773.23 & 3.29 & 1056.47 & 4.49 \\
$\begin{array}{c}\text { construction } \\
\text { land }\end{array}$ & 2090.34 & 8.88 & 2298.01 & 9.77 \\
$\begin{array}{c}\text { Road } \\
\text { Construction } \\
\text { land }\end{array}$ & 160.37 & 0.68 & 290.37 & 1.23 \\
$\begin{array}{c}\text { Artificial } \\
\text { digging pile }\end{array}$ & 341.06 & 1.45 & 372.20 & 1.59 \\
$\begin{array}{c}\text { Desert and } \\
\text { bared earth } \\
\text { Waters } \\
\text { Wetland }\end{array}$ & 380.81 & 1.62 & 372.70 & 1.58 \\
$\begin{array}{c}\text { Total } \\
\text { Table 1. Then }\end{array}$ & 23528.34 & $100.00 \%$ & 23528.34 & $100.00 \%$ \\
\hline
\end{tabular}

Table 1 . The surface cover type of key development zones

The monitoring results show that: the surface cover types of typical key development zones are mainly composed of cultivated land, forest land, garden land, and housing construction land, which accounts for the total area of land cover $87 \%$. The area of cultivated land and forest land reduced significantly from 2010 to 2017, and the area of garden, grassland, housing construction land and construction land increase greatly.

\begin{tabular}{|c|c|c|c|c|}
\hline \multirow{2}{*}{$\begin{array}{l}\text { Vegetation } \\
\text { Types }\end{array}$} & \multicolumn{2}{|c|}{2010} & \multicolumn{2}{|c|}{2017} \\
\hline & $\begin{array}{c}\text { Area } \\
\left(\mathrm{km}^{2}\right)\end{array}$ & $\begin{array}{c}\text { Proportion } \\
(\%)\end{array}$ & $\begin{array}{c}\text { Area } \\
\left(\mathrm{km}^{2}\right)\end{array}$ & $\begin{array}{c}\text { Proportion } \\
(\%)\end{array}$ \\
\hline $\begin{array}{l}\text { Cultivated } \\
\text { land }\end{array}$ & 191.26 & 0.66 & 191.09 & 0.66 \\
\hline Garden land & 1.28 & 0.00 & 1.20 & 0.00 \\
\hline Forest land & 6801.38 & 23.63 & 6801.68 & 23.63 \\
\hline Grass land & 21466.08 & 74.57 & 21324.34 & 74.08 \\
\hline $\begin{array}{c}\text { Housing } \\
\text { construction } \\
\text { land }\end{array}$ & 34.38 & 0.12 & 42.01 & 0.15 \\
\hline Road & 0.84 & 0.00 & 1.57 & 0.01 \\
\hline $\begin{array}{c}\text { Construction } \\
\text { land }\end{array}$ & 7.46 & 0.03 & 11.15 & 0.04 \\
\hline $\begin{array}{c}\text { Artificial } \\
\text { digging pile }\end{array}$ & 5.06 & 0.02 & 7.07 & 0.02 \\
\hline $\begin{array}{l}\text { Desert and } \\
\text { bared earth }\end{array}$ & 156.68 & 0.54 & 152.85 & 0.53 \\
\hline $\begin{array}{l}\text { Waters } \\
\text { Wetland }\end{array}$ & 121.38 & 0.42 & 252.84 & 0.88 \\
\hline Total & 28785.9 & $100.00 \%$ & 28785.8 & $100.00 \%$ \\
\hline
\end{tabular}

Table 2. The land cover type of restricted development zone

The surface cover types of typical key restricted development zone mainly consists of forest land and grassland, which occupy $97.71 \%$ of the total area of the surface coverage. From 2010 to 2017, the area of cultivated land, forest land reduced sharply, while garden land, grassland, housing construction land and structure area were increased greatly.

\begin{tabular}{|c|c|c|c|c|}
\hline \multirow[b]{2}{*}{$\begin{array}{l}\text { Vegetation } \\
\text { Types }\end{array}$} & \multicolumn{2}{|c|}{2010} & \multicolumn{2}{|c|}{2017} \\
\hline & $\begin{array}{l}\text { Area } \\
\left(\mathrm{km}^{2}\right)\end{array}$ & $\begin{array}{c}\text { Proportion } \\
(\%)\end{array}$ & $\begin{array}{l}\text { Area } \\
\left(\mathrm{km}^{2}\right)\end{array}$ & $\begin{array}{c}\text { Proportion } \\
(\%)\end{array}$ \\
\hline $\begin{array}{c}\text { Cultivated } \\
\text { land }\end{array}$ & 5.34 & $0.38 \%$ & 3.73 & $0.27 \%$ \\
\hline Garden land & 0.03 & $0.00 \%$ & 0.04 & $0.00 \%$ \\
\hline Forest land & 918.37 & $65.50 \%$ & 920.78 & $65.67 \%$ \\
\hline Grass land & 153.18 & $10.93 \%$ & 152.26 & $10.86 \%$ \\
\hline $\begin{array}{l}\text { Housing } \\
\text { construction }\end{array}$ & 2.40 & $0.17 \%$ & 2.82 & $0.20 \%$ \\
\hline $\begin{array}{c}\text { Construction } \\
\text { land }\end{array}$ & 0.57 & $0.04 \%$ & 0.75 & $0.05 \%$ \\
\hline $\begin{array}{c}\text { Artificial } \\
\text { digging pile }\end{array}$ & 0.31 & $0.02 \%$ & 0.11 & $0.01 \%$ \\
\hline $\begin{array}{l}\text { Desert and } \\
\text { bared earth }\end{array}$ & 319.59 & $22.79 \%$ & 319.32 & $22.78 \%$ \\
\hline $\begin{array}{c}\text { Waters } \\
\text { Wetland }\end{array}$ & 2.25 & $0.16 \%$ & 2.23 & $0.16 \%$ \\
\hline Total & 1402.05 & $100.00 \%$ & 1402.05 & $100.00 \%$ \\
\hline
\end{tabular}

Table 3. The land cover type of prohibition development zone The cover types of the typical important prohibition development zone mainly consist of forest land, grassland, 
desert and bared earth, which accounts for the total area of land cover $99.31 \%$. From 2010 to 2017, the area of cultivated land, grassland, desert and bared earth decreased greatly, but the area
Land cover transfer matrix is mainly used to show the mutual transformation between land cover types. The statistics of land cover transfer are shown in the following table:

of forest land, housing construction land and structure were increased greatly.

\begin{tabular}{|c|c|c|c|c|c|c|c|c|c|c|c|}
\hline $\begin{array}{l}2017 \\
2010\end{array}$ & $\begin{array}{l}\text { Cultivated } \\
\text { land }\end{array}$ & $\begin{array}{c}\text { Garden } \\
\text { land }\end{array}$ & $\begin{array}{l}\text { Forest } \\
\text { land }\end{array}$ & Grass land & $\begin{array}{c}\text { Housing } \\
\text { construction } \\
\text { land }\end{array}$ & Road & $\begin{array}{c}\text { Construction } \\
\text { land }\end{array}$ & $\begin{array}{l}\text { Artificial } \\
\text { digging } \\
\text { pile }\end{array}$ & $\begin{array}{c}\text { Desert } \\
\text { and } \\
\text { bared } \\
\text { earth }\end{array}$ & $\begin{array}{l}\text { Waters } \\
\text { Wetland }\end{array}$ & Total \\
\hline $\begin{array}{l}\text { Cultivated } \\
\text { land }\end{array}$ & 7596.57 & 802.84 & 142.69 & 231.50 & 177.40 & 48.05 & 110.31 & 133.97 & 2.86 & 54.97 & 9301.15 \\
\hline Garden land & 94.46 & 1497.37 & 31.69 & 39.27 & 29.25 & 7.98 & 12.40 & 28.39 & 0.32 & 2.41 & 1743.55 \\
\hline Forest land & 69.58 & 74.97 & 7582.00 & 87.94 & 32.98 & 7.38 & 5.99 & 28.54 & 16.72 & 8.86 & 7914.96 \\
\hline Grass land & 22.49 & 12.14 & 81.18 & 488.19 & 65.92 & 8.89 & 17.10 & 35.45 & 17.99 & 24.45 & 773.78 \\
\hline $\begin{array}{c}\text { Housing } \\
\text { construction } \\
\text { land }\end{array}$ & 35.33 & 10.38 & 17.51 & 63.49 & 1887.63 & 11.75 & 15.90 & 46.12 & 0.26 & 1.11 & 2089.48 \\
\hline Road & 0.07 & 0.07 & 0.27 & 0.80 & 0.83 & 205.71 & 0.57 & 0.66 & 0.00 & 0.02 & 208.99 \\
\hline $\begin{array}{c}\text { Construction } \\
\text { land }\end{array}$ & 16.52 & 8.00 & 2.36 & 8.16 & 16.89 & 1.51 & 100.46 & 5.64 & 0.06 & 0.87 & 160.47 \\
\hline $\begin{array}{c}\text { Artificial } \\
\text { digging pile }\end{array}$ & 13.63 & 4.76 & 20.21 & 74.32 & 83.51 & 21.05 & 25.40 & 79.54 & 6.74 & 12.58 & 341.73 \\
\hline $\begin{array}{l}\text { Desert and } \\
\text { bared earth }\end{array}$ & 0.87 & 0.20 & 19.67 & 28.93 & 0.31 & 0.20 & 0.43 & 3.01 & 304.39 & 21.48 & 379.49 \\
\hline $\begin{array}{l}\text { Waters } \\
\text { Wetland }\end{array}$ & 11.29 & 2.19 & 2.16 & 31.67 & 3.27 & 2.64 & 1.50 & 9.10 & 23.22 & 527.70 & 614.74 \\
\hline Total & 7860.82 & 2412.92 & 7899.73 & 1054.27 & 2297.98 & 315.14 & 290.07 & 370.41 & 372.55 & 654.44 & 23528.33 \\
\hline
\end{tabular}

Table 4 . The land cover transition matrix of key development zones

The results showed that: The type of surface cover varies greatly; The largest change in arable land occurs, and the main flow is arable land to the garden, house construction land, artificial excavation sites and structures.

\begin{tabular}{|c|c|c|c|c|c|c|c|c|c|c|c|}
\hline $\begin{array}{l}2017 \\
2010\end{array}$ & $\begin{array}{l}\text { Cultivated } \\
\text { land }\end{array}$ & $\begin{array}{c}\text { Garden } \\
\text { land }\end{array}$ & $\begin{array}{c}\text { Forest } \\
\text { land }\end{array}$ & Grass land & $\begin{array}{c}\text { Housing } \\
\text { construction } \\
\text { land }\end{array}$ & Road & $\begin{array}{c}\text { Construction } \\
\text { land }\end{array}$ & $\begin{array}{l}\text { Artificial } \\
\text { digging } \\
\text { pile }\end{array}$ & $\begin{array}{c}\text { Desert } \\
\text { and } \\
\text { bared } \\
\text { earth }\end{array}$ & $\begin{array}{l}\text { Waters } \\
\text { Wetland }\end{array}$ & Total \\
\hline $\begin{array}{l}\text { Cultivated } \\
\text { land }\end{array}$ & 189.41 & 0.00 & 0.05 & 0.33 & 1.22 & 0.00 & 0.11 & 0.09 & 0.00 & 0.02 & 191.25 \\
\hline Garden land & 0.06 & 1.20 & 0.00 & 0.00 & 0.02 & 0.00 & 0.00 & 0.00 & 0.00 & 0.00 & 1.28 \\
\hline Forest land & 0.13 & 0.00 & 6794.63 & 4.62 & 0.07 & 0.02 & 0.04 & 0.29 & 0.77 & 0.87 & 6801.44 \\
\hline Grass land & 1.38 & 0.00 & 6.26 & 21431.67 & 6.81 & 0.69 & 4.16 & 2.18 & 5.75 & 6.87 & 21465.77 \\
\hline $\begin{array}{c}\text { Housing } \\
\text { construction } \\
\text { land }\end{array}$ & 0.09 & 0.00 & 0.00 & 0.57 & 33.68 & 0.00 & 0.03 & 0.03 & 0.00 & 0.00 & 34.41 \\
\hline Road & 0.00 & 0.00 & 0.00 & 0.00 & 0.00 & 0.84 & 0.00 & 0.00 & 0.00 & 0.00 & 0.84 \\
\hline $\begin{array}{c}\text { Construction } \\
\text { land }\end{array}$ & 0.00 & 0.00 & 0.00 & 0.68 & 0.02 & 0.00 & 6.86 & 0.02 & 0.00 & 0.01 & 7.58 \\
\hline $\begin{array}{c}\text { Artificial } \\
\text { digging pile }\end{array}$ & 0.00 & 0.00 & 0.00 & 0.50 & 0.19 & 0.00 & 0.08 & 4.21 & 0.02 & 0.04 & 5.03 \\
\hline $\begin{array}{l}\text { Desert and } \\
\text { bared earth }\end{array}$ & 0.00 & 0.00 & 0.49 & 12.77 & 0.03 & 0.01 & 0.00 & 0.10 & 137.19 & 6.12 & 156.70 \\
\hline $\begin{array}{c}\text { Waters } \\
\text { Wetland }\end{array}$ & 0.00 & 0.00 & 0.32 & 10.95 & 0.00 & 0.01 & 0.00 & 0.16 & 9.14 & 100.90 & 121.48 \\
\hline Total & 191.08 & 1.20 & 6801.75 & 21462.09 & 42.04 & 1.57 & 11.28 & 7.07 & 152.87 & 114.82 & 28785.77 \\
\hline
\end{tabular}

Table 5. The land cover transition matrix of restricted development zone 
The results showed that: The total conversion of surface cover in the Zoige Grassland Wetland Ecological Function Zone is
$85.18 \mathrm{~km}^{2}$. Generally, the transfer between different types of land is small, and the land cover type is stable.

\begin{tabular}{|c|c|c|c|c|c|c|c|c|c|c|}
\hline $\begin{array}{l}2017 \\
2010\end{array}$ & $\begin{array}{l}\text { Cultivated } \\
\text { land }\end{array}$ & $\begin{array}{c}\text { Garden } \\
\text { land }\end{array}$ & Forest land & Grass land & $\begin{array}{l}\text { Housing } \\
\text { construction }\end{array}$ & $\begin{array}{l}\text { Construction } \\
\text { land }\end{array}$ & $\begin{array}{l}\text { Artificial } \\
\text { digging } \\
\text { pile }\end{array}$ & $\begin{array}{l}\text { Desert and } \\
\text { bared earth }\end{array}$ & $\begin{array}{c}\text { Waters } \\
\text { Wetland }\end{array}$ & Total \\
\hline $\begin{array}{c}\text { Cultivated } \\
\text { land }\end{array}$ & 3577206.12 & 11812.08 & 1339605.34 & 209741.72 & 152896.59 & 5507.93 & 21467.40 & 0.00 & 21539.95 & 5.34 \\
\hline Garden land & 0.00 & 31431.50 & 0.00 & 0.00 & 0.00 & 0.00 & 0.00 & 0.00 & 0.00 & 0.03 \\
\hline Forest land & 49897.38 & 0.00 & 915539702.90 & 2676304.52 & 47291.94 & 9602.62 & 3296.87 & 45471.26 & 0.00 & 918.37 \\
\hline Grass land & 80008.64 & 0.00 & 3687704.43 & 148976353.30 & 132994.51 & 188453.00 & 18361.34 & 97135.87 & 2573.72 & 153.18 \\
\hline $\begin{array}{l}\text { Housing } \\
\text { construction }\end{array}$ & 15828.51 & 0.00 & 6263.78 & 21164.32 & 2354165.17 & 649.76 & 0.00 & 0.00 & 0.00 & 2.40 \\
\hline $\begin{array}{c}\text { Construction } \\
\text { land }\end{array}$ & 0.00 & 0.00 & 7019.97 & 11939.35 & 22438.24 & 525640.11 & 0.00 & 0.00 & 0.00 & 0.57 \\
\hline $\begin{array}{c}\text { Artificial } \\
\text { digging pile }\end{array}$ & 2123.59 & 0.00 & 12083.96 & 100418.72 & 106691.51 & 20712.41 & 70583.40 & 0.00 & 0.00 & 0.31 \\
\hline $\begin{array}{l}\text { Desert and } \\
\text { bared earth }\end{array}$ & 0.00 & 0.00 & 187350.29 & 253967.13 & 0.00 & 0.00 & 0.00 & 319146747.40 & 0.00 & 319.59 \\
\hline $\begin{array}{l}\text { Waters } \\
\text { Wetland }\end{array}$ & 0.00 & 0.00 & 0.00 & 13473.59 & 0.00 & 0.00 & 0.00 & 33791.25 & 2207170.54 & +2.25 \\
\hline Total & 3.73 & 0.04 & 920.78 & 152.26 & 2.82 & 0.75 & 0.11 & 319.32 & 2.23 & 1402.05 \\
\hline
\end{tabular}

Table 6 . The land cover transition matrix of prohibition development zone

The results showed that: The transitions between land cover types are more frequent, mainly reflected in the conversion between forest land, farmland, and grassland.

\section{CONCLUSIONS}

Based on the characteristics of different main functional areas, the paper selects the typical main functional areas in Sichuan Province and uses the two-phase remote sensing image ZY-3 with better than $2.5 \mathrm{~m}$ resolution to extract the ground cover type data, and combines the basic geographic information data and various professional statistical data. , carry out targeted monitoring and statistics and analysis. The scope of monitoring of the selected main functional areas was about $57000 \mathrm{~km}^{2}$ which is typical. Among them, the typical key development zone is $24000 \mathrm{~km}^{2}$, the typical restricted development zone is $28700 \mathrm{~km}^{2}$, and the typical banned development zone is about 0.4 million $\mathrm{km}^{2}$. The research results can provide data support for ecological and environmental protection.

\section{ACKNOWLEDGEMENTS}

Thanks are due to Sichuan science and technology plan key research and development project (NO. 18ZDYF2292).

\section{REFERENCES}

Liu, J., 2016a. Remote Sensing-based analysis of the spatiotemporal characteristics of built-up area across China based on the Plan for major function-oriented zones. Acta Geographica Sinica, 71(3), pp. 355-369.

Fan, J., 2015a. Draft of major function oriented zoning of China. Acta Geographica Sinica, 70(2), pp. 186-201.
Pei, W., 2010a. Research on the appraisals and countermeasures of the disaster risks of Sichuan tourism places evoked by the earthquake. Soft Science, 24(4), pp. 89-93.

Wang, G. X., Liu, T., 2017a. Coupling relationship change between urbanization and eco-environment of resource-based cities in Central China. China Population, Resources and Environment, 27(7), pp. 80-88.

Zhang, L. Q., Cai, J. M., 2010a. Urbanization development characteristics and its path selection process in fragile ecological environment. Ecology and Environmental Sciences, 19(11), pp. 2764-2772.

Wang, S. Y., Zhang, X. X., Zhu, T., 2016a. Assessment of ecological environment quality in the Changbai Mountain Nature Reserve based on remote sensing technology. Progress in Geography, 35(10), pp. 1269-1278.

Sun, D. Q., Jiang, J. L., Xu, J. B., et al., 2015a. Space-time analysis of the changing patterns of population pressure on the ecological environment in China. Chinese Journal of Population, Resources and Environment, 13(4), pp. 341-348.

Nie, Y., Zhang, Y. L., Liu, L. S., et al., 2010a. Monitoring glacier change based on remote sensing in the Mt. Qomolangma national nature preserve, 1976-2006. Acta Geographica Sinica, 65(1), pp. 13-28.

Li, G. Q. Li, X. B. Li, G. M., et al., 2013a. Comparison of spectral characteristics between China HJ1-CCD and Landsat 5 TM imagery. IEEE Journal of Selected Topics in Applied Earth Observations \& Remote Sensing, 6(1), pp. 139-148. 